[3] Shafi, M.; Moore, D.: Adaptive Equalizer Improvements for 16QAMI and 64QAM Digital Radio. IEEE Int. Conf. on Comm. ICC 1984, S. 998-1002

[4] Schmidt, W.: Grundlagen für die Anwendung des Orthogonalfilters zur Filternng uned Formung von Signalen unter besonderer Perïcksichtignng der Entzerrung von Datenïbertragungskanälep, Universität Karkruhe, 1979

[5] Camphell, J. C.; Coutts, R. P.: Outage Prediction of Digital Radio Systems. Electron. Lett. 18 (1982) 25, S. 1071 - 1072

[6] Leclert, A.; Vandamme, P.: Timing Synchronization in Digital Radio Systerns with Mnltipath Propagotion IEEE Int. Conf. on Comm ICC 1983, S. B6.6.1-5.

[7] Herbig. P.: Adaptive Time Domain Equalization for LOS Channeks. Euron Conf. on Radio-Relay Syst. ECRR 1986, S. 182-189.

[8] Gitlin, R. D.; Weinstein, S. B.: Fractionally-Spaced Equalization: An Improsed Digital Transversal Equalizer. Bell Syst. Tech. J. 60 (1981) 2, S. 275-296.

[9] Haykin, S.: Adaptive Filter Theory. Englewood Ciffis, New Jersey: Prentice-Hall, 1986.
[10] Glauner, M.: Symbol- and Bitfehlerwahrscheinlichkeit von M-QAM im idealen Nyquist-Kanal mit additivem ganfschem Rauschen. Arch. elektr. Cbertragung 37 (1983) $3-4,5.123-129$

[11] Gitlin, R. D.; Meadors, H. C. JR.; Weinstein, S. B.: The Tap-Leakage Algorithm: An Algorithm for the Stable Operation of a Dipitally Implemented, Fractionally Spaced Adaptive Equalizer. Bell Syst. Tech. J. 61 (1982) 8, S. 1817-1839.

[12] Ingerboeck, G.: Fractional Tan-Spacing Equalizer and Consequences for Clock Recovery in Data Modems IE.EE Trans, on Comm. COM1-24(1976) 8, S. 856-864.

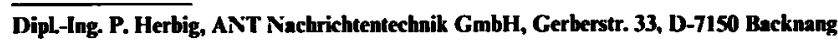
(Fingegangen am 8.3.1988)

\title{
Abklingverhalten des Taktsignals bei Einsatz von SAW- Transversalfiltern zur Taktsynchronisation in digitalen Regeneratoren
}

\author{
Deexcitation Behavior of the Timing Wave in Digital Regenerators with SAW Transversal Filters \\ for Timing Recovery
}

Von Winfried Guba

Mitteilung aus dem Instilut für Hochfrequenztechnik der UNIVERSITÄT IIANNOVER

Übersicht:

Das Abklingverhalten des Taktsignals am Ausgang des in einer Taktgewinnungseinrichtung eingesetzten SAW-Transversalfilters wird auf einfache Weise analytisch beschrieben. Damit ist eine rasche Abschätzung des für den nachfolgenden Begrenzerverstärker benötigten Dynamikbereichs bzw. der maximal zulässigen Folge gleicher Symbole $\left(, 00^{\circ}\right.$ oder NRZ ,1“) innerhalb des empfangenen Datenstroms möglich. Das Ergebnis ist in guter Ubereinstimmung mit Meßergebnissen, zeigt aber erhebliche Abweichungen zu den bisher in der Literatur angegebenen theoretischen Resultaten; die Gründe hierfür werden diskutiert.

Abstract:

This paper presents a simple analytical expression for the deexcitation behaviour of the timing wave at the output of a SAW transversal filter inserted in a timing recovery circuit, which allows for a quick estimation of the dynamic range of the postfilter limiting amplifier respectively the longest allowable transitionless string (" 0 " or NRZ " 1 ") within the received data signal. The result is in good agreement with measurements, but deviates considerably from those given in the literature; the reasons will be discussed.

Für die Dokumentation:

Taktgewinnung / SAW-Transversalfilter / Abklingverhalten

\section{Einleitung}

In den letzten Jahren hat die Bedeutung von SAWTransversalfiltern für den Einsatz in digitalen Übertragungssystemen stark zugenommen, da sie insbesondere bei hohen Bitraten vorteilhaft gegenüber PLL-Schaltungen eingesetzt werden können [1-4].

Die Taktgewinnungseinrichtung in einem Regenerator (Bild 1a) hat die Aufgabe, aus dem Detektionssignal $x(t)$ die für den Detektor nötige Taktinformation, die in $z(t)$ enthalten ist, $\mathrm{zu}$ gewinnen. Im allgemeinen ist hierzu eine nichtlineare Vorverzerrung (Vorfilter, NL1) nötig, um im Spektrum von $y(t)$ eine diskrete Komponente bei der Taktfrequenz zu erzeugen (Bild 1b). Das Taktfilter (z.B. SAW-Filter oder PLL-Schaltung) dient dazu, diese Spektralkomponente auszufiltern und damit den Impulsgenerator NL 2 anzusteuern, der dem Detektor das Taktsignal $z(t)$ in Form einer möglichst äquidistanten Impulsfolge liefert. Liegt im Fall einer ungünstigen Datenfolge (Folge von „0“ oder NRZ „1“) am Eingang des Taktfilters für längere Zeit eine konstante Spannung vor, so fällt die Ausgangsamplitude bei Einsatz eines SAW-Filters mehr und mehr ab, so daB unter Umständen ein Verlust der Synchronisation auftreten kann. Aus diesem Grund wird häufig ein Begrenzerverstärker eingesetzt, der diesen Effekt innerhalb seines Dynamikbereichs kompensieren kann; in [1-3] werden Berechnungsmöglichkeiten aufgezeigt, um den Dynamikbereich in Abhängigkeit von der Güte des SAW-Transversalfilters zu bestimmen.

Im folgenden wird gezeigt, daß die in der Literatur vorgestellten Ergebnisse deutlich zu pessimistisch ausfallen. Hierzu wird in quantitativer Übereinstimmung mit Meßergebnissen eine analytische Beschreibung des Leistungsab- 

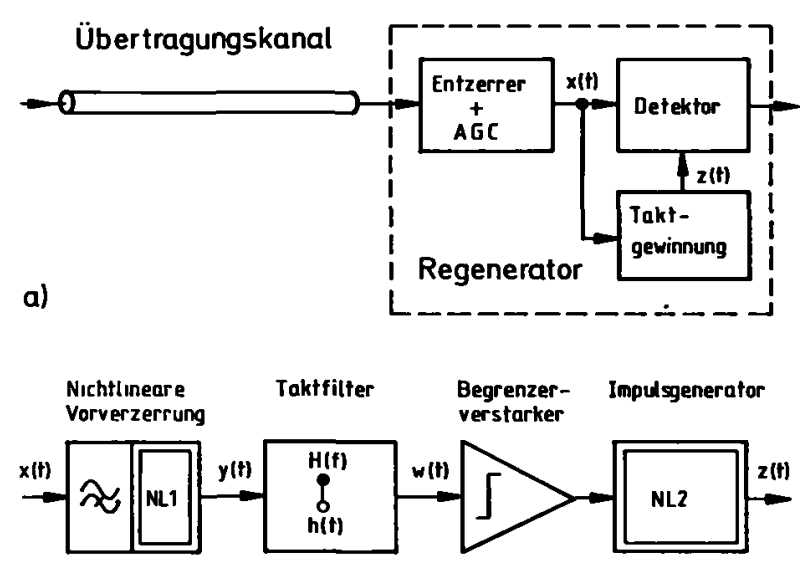

b)

Bild 1: Blockschaltbild eines Regenerators (a) und der zugehörigen Taktgewinnungseinrichtung (b)

falls am Ausgang des SAW-Transversalfilters angegeben. Bei Vorgabe der im System maximal möglichen übergangslosen Zeit kann so direkt der benötigte Dynamikbereich des nachfolgenden Begrenzerverstärkers bzw. bei Vorgabe des Dynamikbereichs die maximal zulässige übergangslose Zeit abgeschätzt werden.

\section{Abklingverhalten des Taktsignals}

Im praktischen Anwendungsfall wird eine starke Fehlanpassung zwischen dem akustischen Strahlungsleitwert $G_{\mathrm{a}}(f)$ des SAW-Filters bei der Mittenfrequenz $f_{\mathrm{c}}=1 / T$ und dem Leitwert $G_{L}$ der äußeren Beschaltung angestrebt, d.h.

$$
\mu=G_{\mathrm{a}}\left(f_{\mathrm{c}}\right) / G_{\mathrm{L}} \ll 1 \text {. }
$$

Hierdurch werden unerwünschte Mehrfachreflexionen (Triple-Transit-Signal) zwischen den Interdigitalwandlern bzw. ,jitter peaking"-Effekte vermieden [4]. und die Einhüllende der Stoßantwort $h(t)$ des SAW-Transversalfilters verläuft entsprechend Bild 2 dreieckförmig [5]. Durch die Anzahl $n$ der Perioden der akustischen Oberflächenwelle zwischen den Wandlern ist die Verzögerungszeit $n / f_{c}=n T$ festgelegt, nach der am Ausgang des zweiten Interdigitalwandlers die Reaktion beginnt. Mit Hilfe der Anzahl $N_{\mathrm{T}}$ der Fingerpaare pro Wandler bzw. der Filtergüte

$$
Q \simeq \frac{\pi}{2} N_{\mathrm{T}} \quad \text { für } \quad \mu \ll 1
$$

kann dann der Zeitpunkt $\left(n+N_{\mathrm{T}}\right) T$ bestimmt werden, zu dem die Stoßantwort ihren Maximalwert erreicht.

Die im folgenden durchgeführte Berechnung beruht auf der Überlegung, daß bei Anregung des SAW-Transversalfilters (Schmalbandfilter) durch eine Pulsfolge $y(t)$ das Ausgangssignal $w(t)$ im wesentlichen durch den taktfrequenten Anteil dieser Folge bestimmt wird. Durch eine Transformation in den Tiefpaßbereich wird die Berechnung von $w(t)$ vereinfacht; als Erregung ergibt sich dann entsprechend Bild 3 ein Rechteckimpuls, der genügend lang sein muß, um ein vollständiges Einschwingen zu gewährleisten.

Die Anwendung der Faltungsoperation

$$
w_{\mathrm{TP}}(t)=h_{\mathrm{TP}}(t) * y_{\mathrm{TP}}(t)=\int_{0}^{t} y(t-\tau) h(\tau) \mathrm{d} \tau
$$

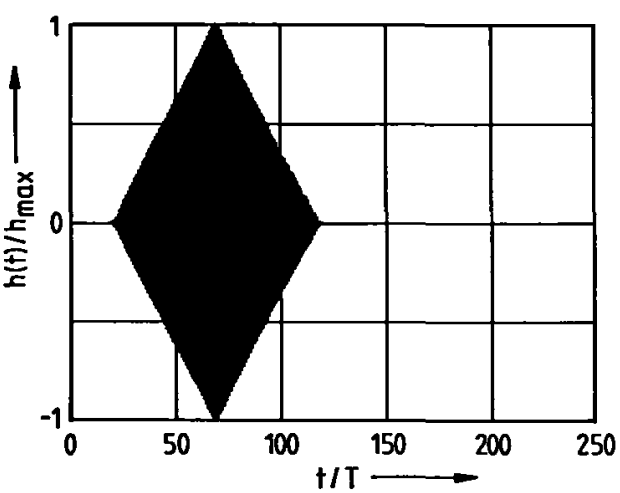

Bild 2: Stoßantwort $h(t)$ für $\mu=0,01, N_{\mathrm{T}}=50$ und $n=20$

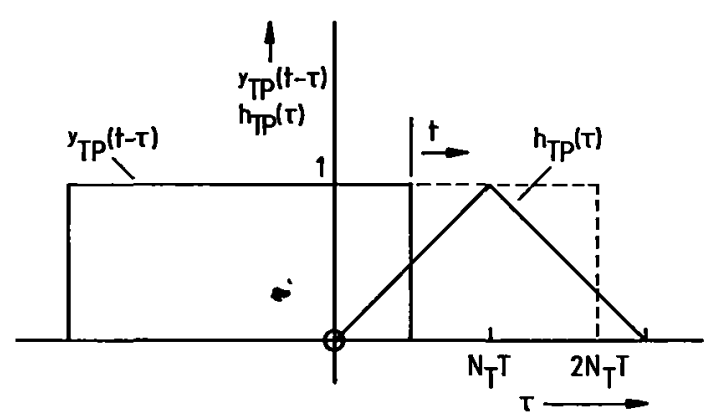

Bild 3: Faltung im Tiefpaßbereich

ergibt für den Einschwingvorgang (Beginn bei $t=0$ )

$w_{\mathrm{TP}}^{\mathrm{e}}(t)=\left\{\begin{array}{lll}\frac{t^{2}}{2 N_{\mathrm{T}} T} & \text { für } & 0<\mathrm{t} \leqq \mathrm{N}_{\mathrm{T}} T, \\ -N_{\mathrm{T}} T+2 t-\frac{t^{2}}{2 N_{\mathrm{T}} T} & \text { für } & N_{\mathrm{T}} T<t \leqq 2 N_{\mathrm{T}} T, \\ N_{\mathrm{T}} T & \text { für } & t>2 N_{\mathrm{T}} T .\end{array}\right.$

Für den Abklingvorgang (Beginn bei $t=0$ ) erhält man

$w_{\mathrm{TP}}^{\mathrm{a}}(t)=\left\{\begin{array}{lll}N_{\mathrm{T}} T-\frac{t^{2}}{2 N_{\mathrm{T}} T} & \text { für } & 0<t \leqq N_{\mathrm{T}} T, \\ 2 N_{\mathrm{T}} T-2 t+\frac{t^{2}}{2 N_{\mathrm{T}} T} & \text { für } & N_{\mathrm{T}} T<t \leqq 2 N_{\mathrm{T}} T, \\ 0 & \text { für } & t>2 N_{\mathrm{T}} T .\end{array}\right.$

Der Leistungsabfall nach Abschalten der Erregung berechnet sich mit

$$
w^{\mathrm{a}}(k T)=w_{\mathrm{TP}}^{\mathrm{a}}(k T), k=1,2,3, \ldots
$$

wie folgt:

$$
D(k T) / \mathrm{dB}=10 \log \left(\frac{n^{\mathrm{a}}(k T)}{w^{\mathrm{a}}(0)}\right)^{2} .
$$

Nach Einführung der normierten Zeit

$$
z=\frac{k}{N_{\mathrm{T}}} \sim \frac{\text { Zeit }}{\text { Filtergüte }}
$$

ergibt sich

$D(z) / \mathrm{dB}=\left\{\begin{array}{lll}20 \log \left(1-0,5 z^{2}\right) & \text { für } & 0<z \leqq 1, \\ 20 \log 2(1-0,5 z)^{2} & \text { für } & 1 \leqq z<2, \\ -\infty & \text { für } & z \geqq 2 .\end{array}\right.$ 


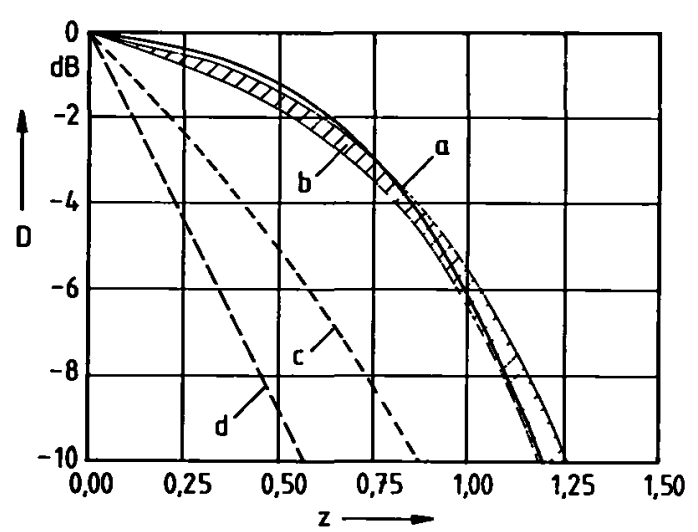

Bild 4: Leistungsabfall nach Abschalten der Erregung (a) nach (7), (b) Messung, (c) nach (9) gemäB [3], (d) nach (8) gemäß $[1,2]$

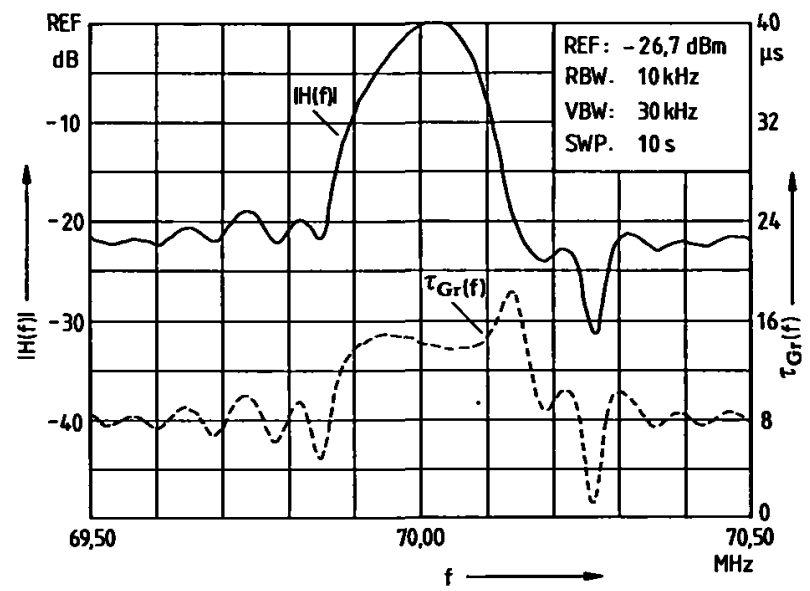

Bild 5: Gemessene Übertragungsfunktion des verwendeten SAWBandpasses (BP 70-60-5-118 A von Andersen Laboratories)

$|H(f)|$ Betrag der Übertragungsfunktion

$\tau_{\mathrm{Gr}}(f)$ Gruppenlaufzeit

$R E F$ Bezugspegel

$R B W$ Bandbreite des ZF-Filters

$V B W$ Videobandbreite

$S W P$ Sweep

In Bild 4 sind der Leistungsabfall $D(z)$ bzw. der benötigte Dynamikbereich $A=1 / D$ des Begrenzerverstärkers nach (7) sowie die aus der Literatur [1-3] bekannten Ergebnisse dargestellt. Der in [6] für einen Resonanzkreis angegebene Leistungsabfall

$$
D(z) / \mathrm{dB}=20 \log \mathrm{e}^{-2 z}
$$

führt bei Verwendung eines SAW-Transversalfilters zu einer drastischen Fehleinschätzung von $D(z)[1,2]$, da es sich in diesem Fall um ein nichtminimalphasiges Filter mit zeitlich begrenzter Stoßantwort handelt. In [3] wird der Charakter des SAW-Filters durch das $\delta$-Funktionen-Modell berücksichtigt; dennoch zeigt der dort berechnete Verlauf

$$
D(z) / \mathrm{dB}=20 \log (1-0,5 z)^{2}
$$

im Vergleich zu (7) ebenfalls deutliche Abweichungen, da (9) nur im Bereich $1 \leqq z<2$ Gültigkeit besitzt (s. (7)). Es findet aber schon im Bereich $0<z \leqq 1$ ein Leistungsabfall entsprechend (7) statt; dies kann auch auf einfache Weise mit dem $\delta$-Funktionen-Modell gezeigt werden.
Eine zum Vergleich mit (7) durchgeführte numerische Simulationsrechnung unter Zugrundelegung der Übertragungsfunktion des SAW-Transversalfilters nach [4] und bei Erregung des Filters durch eine RZ-Pulsfolge zeigt auch bei Variation von $\mu(\mu \leqq 0,35)$ und $n$ (hierdurch wird neben der Verzögerungszeit auch der u.U. unsymmetrische Rippel im Durchlaßbereich beeinfluBt [4]) derart geringe Abweichungen, da $B$ auf eine getrennte Darstellung verzichtet wird. Der benötigte Dynamikbereich $\boldsymbol{A}$ des Begrenzerverstärkers stellt sich nach (7) bei einer übergangslosen Folge von $k$ Symbolen in Abhängigkeit von der Güte $Q$ des SAW-Transversalfilters wie folgt dar:

$$
A=-20 \log \left[1-\frac{1}{2}\left(\frac{k \pi}{2 Q}\right)^{2}\right] \text { für } Q \geqq \frac{\pi}{2} k .
$$

\section{Messungen}

Die Ergebnisse (7) bis (9) unterscheiden sich sehr stark, so daß eine Entscheidung hinsichtlich ihrer Brauchbarkeit durch Messungen getroffen werden muBte. Der Leistungsabfall $D(z)$ am Ausgang eines durch eine periodische Folge von RZ-Bursts erregten SAW-Transversalfilters mit der gemessenen Übertragungsfunktion gemäß Bild 5 wurde mit Hilfe eines Oszilloskops bestimmt; unter Berücksichtigung der Ablesegenauigkeit ([5], S. 115) erhält man den in Bild 4 (Kurve b) gezeigten Bereich für die Meßwerte. Ebenso wie bei der Simulation zeigte sich auch hier, daß der Einfluß der Anpassung vernachlässigbar ist ([5], S. 116). Die experimentellen Ergebnisse werden also von der hier abgeleiteten Gleichung (7) im Gegensatz zu (8) und (9) gut beschrieben.

\section{Zusammenfassung}

Es wird ein analytischer Ausdruck für den benötigten Dynamikbereich eines Begrenzerverstärkers angegeben, der zur Kompensation des Leistungsabfalls bei übergangslosen Datenfolgen in einer Taktgewinnungseinrichtung hinter dem SAW-Taktfilter eingesetzt wird.

Die theoretischen Ergebnisse gemä $B$ (7) stimmen gut mit Meßwerten sowie den durchgeführten Simulationsrechnungen überein. Ein Vergleich mit den bisherigen Angaben aus der Literatur zeigt, daß sich dort aufgrund unzulässiger Vereinfachungen $[1,2]$ bzw. einer unvollständigen Beschreibung des Abklingvorgangs [3] deutlich zu pessimistische Werte ergeben.

Literatur:

[1] Rosenherg, R. I.; Ross, D. G.; Trischitta, P. R.; Fishman, D. A.; Armilage, C. B. Optical Fiber Repeatered Transmission Systems Utilizing SAW Filters. IEEE Trans. on Sonics and Liltrasonies, Vol. 30 (1983) S. 119-126.

[2] Armitage, C. B.: SAW Filter Retiming in the AT \& T $432 \mathrm{Mb} / \mathrm{s}$ Lightnave Regenerafor. Proc. 10th ECOC, Sept. 1984, S. 102-103.

[3] Rosenberg, R. L.; Chamzas, C.; Fishman, D. A.: Timing Recovery with SAW Transversal Filters in the Regenerators of Lindersea Long-Hanl Fiber Transmiscien Systems. IEEE J. Selected Areas in Communications, Vol. SAC-2 (1984) S.957-965.

[4] Fishman, D. A.; Rosenberg, R. L.; Chamzas C.: Analysis of Jitter Peaking Effeets in Digital Long-Hanl Transmission Systems Using SAW-Filter Retiming. IEEE Trans. on Communications, Vol, COM-33 (1985) S. 654-664.

[5] Lemke, R.: Einschwingverhalten von Tahtregeneratoren mit SAW-Bandpüssen. Diplomarbeit, Cniversitat Hannover, 1987.

[6] Inowe, H.; Sakamoto, T.; Kasai, H.; Yamaguchi, H.: PCM-400 M Regenerative Repeater and Performance. Rev. Elec. Commun. Lab., NTT Public Corp. Vol. 24 (1976) S. 699-710. Dipl-Ing. W. Guba, Institut fir Hochfrequenztechnik, Lniversitant Hannover,
Appekstr.9A, D-3000 Hannover 1

(Eingegangen am 5. 10. 1987, ت̈herarbeitete Version am 15.1. 1988) 